\title{
Quand on ne peut croire ce que l'on voit Stratégies énonciatives et manipulations perceptives dans les œuvres d'Alexa Meade
}

\author{
Audrey Moutat \\ Université de Limoges, laboratoire CeReS (FR) \\ e-mail : audrey.moutat@yahoo.fr
}

\section{Résumé}

Cet article propose une étude des dispositifs de l'énonciation visuelle en jeu dans la production d'œuvres d'art au statut médiatique complexe. Il s'agira de confronter les traces manifestées au sein de ces œuvres à leur saisie perceptive par un énonciataire, instance qui perçoit et interprète ce qu'il appréhende par ses sens.

De ce fait, nous proposons de nous tourner vers les « formes encore imprégnées du faire », de considérer la production textuelle de l'œuvre et de la faire dialoguer avec les mécanismes perceptifs générés par les traces du faire productif. C'est ainsi que nous envisageons de mettre en évidence la dynamique de la sémiose perceptive suscitée par de telles œuvres.

\begin{abstract}
This article studies the features of the visual enunciation involved in the production of works of art with a complex media status. It will confront the traces manifested within these works to their perceptual input by an enunciatee, an instance that perceives and interprets what he learns from the senses.

Therefore, we propose to turn to the "forms still impregnated by the doing," to consider the textual production of the work and make it dialogue with perceptual mechanisms generated by the footsteps of the productive doing. So we aim at highlighting the dynamics of the perceptive semiosis aroused by such works.
\end{abstract}

\section{Mots-clefs}

Énonciation visuelle - Sémiose perceptive - Intermédiation - Textualité de l'image Manipulation énonciative

\section{Keywords}

Visual enunciation - Perceptive semiosis - Intermediation - Textuality of images Enunciative manipulation

\author{
Sommaire \\ Préambule \\ 1. Présentation du corpus \\ 2. De la texture à l'œuvre \\ 3. Une œuvre intermédiatique \\ 4. La manipulation énonciative de l'œuvre d'Alexa Meade \\ Bibliographie
}


Audrey Moutat | Quand on ne peut croire ce que l'on voit

\section{Préambule}

Cette analyse se fonde sur la volonté de questionner le concept d'énonciation en remontant à ses fondamentaux Benvenistiens (1966) et Courtésiens (1991) ; l'objectif étant de les mettre en difficulté, ou plutôt de repousser leurs limites, au regard d'un objet d'analyse complexe : les œuvres d'art d'une jeune artiste américaine, Alexa Meade. Le choix de ce corpus n'est pas anodin. Il permet en effet de problématiser le concept d'énonciation à travers les différents statuts que peut adopter l'actant sujet, entendu non seulement comme une instance qui produit l'énoncé, mais aussi celle qui perçoit et interprète l'objet visuel ainsi proposé (sachant que ces instances peuvent être recouvertes par un seul et même actant).

Instance qui perçoit car rappelons que si l'énonciation a été définie comme une mise en fonctionnement de la langue par l'usage de son plan de l'expression - ce dernier visant une signification propre à l'individu qui l'exploite -, ses frontières se sont élargies sous l'impulsion des travaux de Jean-François Bordron (2002, 2009, 2011) pour qui l'énonciation englobe la perception et la sensation. Alors définie comme un « art de la prise » (2002) produisant une cristallisation des expériences énonciatives et perceptives, l'énonciation se situe en deçà de la mise en fonction de la langue, dans l'immanence même de la perception (autrement dit dans la relation qui unit source et cible de l'acte perceptif).

Ainsi, notre démarche propose de mettre au jour les dispositifs de l'énonciation visuelle en jeu dans les œuvres d'Alexa Meade, à partir des traces manifestées sur leur plan de l'expression, afin de les confronter, dans un second temps, à la perception effective de ces œuvres par un énonciataire, sujet percevant et pensant. Il s'agit de se tourner vers les « formes encore imprégnées du faire » énonciatif, de considérer la production textuelle et de la faire dialoguer avec les mécanismes perceptifs suscités par les marques de cette énonciation dans le discours ; le tout permettant de mettre en exergue la dynamique de la perception et les effets de sens associés.

\section{Présentation du corpus}

Le corpus que nous convoquons se compose d'une sélection ${ }^{1}$ de quelques œuvres d'Alexa Meade, artiste américaine dont le travail se situe à la croisée de la peinture, de la photographie, de l'installation et de la performance. La

1 Bien que l'artiste se soit auparavant essayée aux natures mortes, nous avons décidé de soumettre à l'analyse uniquement les portraits réalisés à même les sujets vivants « re-présentés ». L'ensemble des œuvres citées dans cet article sont accessibles sur le portfolio de l'artiste : <http://www.alexameade.com/> 
Audrey Moutat | Quand on ne peut croire ce que l'on voit

particularité de sa démarche artistique réside dans le choix du support. En effet, l'artiste ne peint pas sur des toiles mais directement sur les sujets que ses œuvres sont censées représenter. Ces sujets se situent dans un décor luimême peint, le tout étant saisi par l'empreinte photographique.

Ce que je fais dans mon art, c'est que je me passe entièrement de la toile ; et si je veux peindre votre portrait, je le peins sur vous, physiquement sur vous. [...] Tout ce qui est dans cette scène, la personne, les vêtements, les chaises, le mur, est couvert par une couche de peinture qui reproduit ce qui est juste en-dessous et, de cette façon, je suis en mesure de partir d'une scène en trois dimensions et de la faire ressembler à une peinture en deux dimensions. Je peux la photographier sous tous les angles et elle aura toujours l'air d'être en 2D. [...] Vous vous demandez peut-être comment j'en suis venue à cette idée de transformer les gens en peinture. Il s'agissait d'ombres. J'étais fascinée par l'absence de lumière et je voulais trouver un moyen de lui donner de la matérialité et de la fixer avant qu'elle ne bouge. J'ai eu l'idée de peindre les ombres. J'ai aimé le fait que je puisse cacher à l'intérieur de cette ombre ma propre version peinte. Et elle y serait presque invisible, jusqu'au moment où la lumière changerait. Et tout d'un coup, mon ombre serait portée à la lumière. ${ }^{2}$

Dès lors, une même œuvre peut recouvrir plusieurs statuts :

1. Le statut d'une peinture : les sujets et objets trouvés sont peints avec de la peinture acrylique mêlée à « une dizaine d'ingrédients, de produits de beauté ou de cuisine, pour que l'ensemble ne sèche pas trop vite et reste plus souple quand le modèle bouge. On évite ainsi les craquelures »3. L'artiste peint ainsi " sur les gens d'une façon qui les transforme en tableaux en deux dimensions. [Elle] pein[t] les ombres telles qu'elles existent dans la vraie vie, directement sur eux ${ }^{4}$. Elle commence par le fond et les vêtements que le modèle portera ; puis c'est au tour du visage et des parties du corps visibles du sujet.

2. Le statut d'une performance : lors de ses expositions en galerie, l'artiste se livre souvent à la création d'une œuvre en direct, devant le public. Tel est le cas de la série de séances de portraits réalisée par l'artiste du 17 au 29 mars 2015 dans différents espaces parisiens. Sous les yeux des visiteurs, Alexa Meade applique de la peinture sur ses modèles avant de les immortaliser en photographie.

3. Le statut d'une installation : une fois réalisées, ces " peintures-performances » deviennent des installations qui, exposées en galerie ${ }^{5}$ ou en pleine

2 Traduction de la conférence d'Alexa Meade au TEDGlobal 2013 ( conférence annuelle qui célèbre l'ingéniosité humaine en explorant les idées, l'innovation et la créativité de tous les coins du monde ») disponible à l'adresse suivante : <https:// www.ted.com/talks/alexa_meade?language $=\mathrm{fr}>$.

3 Citation d'Alexa Meade dans un article du journal Le soir, Les tableaux vivants d'Alexa, disponible à l'adresse suivante : <http://archives.lesoir.be/les-tableauxvivants-d-8217-alexa_t-20110829-01K192.html>.

4 Traduction de la conférence d'Alexa Meade au TEDGlobal 2013 ( conférence annuelle qui célèbre l'ingéniosité humaine en explorant les idées, l'innovation et la créativité de tous les coins du monde ») disponible à l'adresse suivante : <https:// www.ted.com/talks/alexa_meade?language $=$ fr $>$

5 Natura morta (2009). 
Audrey Moutat | Quand on ne peut croire ce que I'on voit

rue $^{6}$, modélisent la perception des spectateurs en façonnant leur manière d'appréhender les relations spatiales. C'est ainsi que les frontières entre réalité et représentations se trouvent brouillées. En effet, l'artiste propose parfois d'extraire ses personnages du fond peint pour les intégrer dans l'environnement réel (rue, métro... $)^{7}$. « Les personnes peintes dans l'environnement, dit-elle, fonctionnent sans arrière-plan mais cela participe aussi beaucoup de l'effet quand on voit le personnage sortir du tableau ».

4. Le statut d'une photographie ${ }^{8}$ : diverses réalisations de l'artiste sont ensuite capturées par l'empreinte photographique. Les images ainsi créées constituent les œuvres d'art définitives exposées en galerie et recensées dans le portfolio de l'artiste : "Quand je peins une personne pour une exposition, ou un événement, évidemment l'œuvre d'art rentre chez elle à la fin de la journée pour se doucher et tout disparaît. Et donc je prends beaucoup de photos pour saisir l'esprit d'une œuvre d'art et, de cette façon, elle sera toujours vivante »9.

L'étude que nous proposons portera sur les photographies sélectionnées par l'artiste dans la composition de son portfolio. Le contexte de présentation de l'œuvre que nous retenons pour notre analyse est celui de l'exposition en galerie, contexte pour lequel ces photographies ont finalement été créées. Photographies des installations, et non des performances, elles ne trahissent pas la technique de l'artiste mais interpellent, sur le mode de la tromperie visuelle, le spectateur ${ }^{10}$. À cet égard, la nature de ces images pose déjà un premier problème en ce sens où elle interroge la nature de l'œuvre elle-même : doit-on la considérer comme une photographie ? Une peinture ? L'image d'un objet qui, en l'occurrence, serait une installation éphémère ? Ou bien s'agit-il de la conjonction des trois ? C'est ce que nous tâcherons de comprendre à la suite de cet article.

Du portrait, Nelson Goodman (2005) affirme qu'il ne peut être une copie de l'autre ; c'est une énonciation qui construit un effet de sens sur le modèle-source. Reste à comprendre comment l'instance énonçante parvient à réguler cette présence de l'autre.

Les images ${ }^{11}$ d'Alexa Meade proposent une négation du rapport intersubjectif, pouvant même aller jusqu'à la négation identitaire du sujet représenté. Ce dernier se refuse à l'autre en le fuyant du regard par sa pose de profil ou de trois-quarts face. Quant aux rares fois où il se présente de face, il baisse ou

6 Installation pour la mini cooper à Tokyo.

7 Transit, 3 (2009).

8 Notons que si certaines œuvres sont exposées comme installations, la plupart d'entre elles sont des œuvres photographiques.

9 Alexa Meade (Ibid.)

10 Nous soulignons également que ces images, initialement diffusées via les médias sociaux (portfolio en ligne de l'artiste, Flickr, Instagram) sont rapidement devenues virales : relayées par la presse en ligne, elles ont largement été diffusées sur les réseaux sociaux et touchent un large public, non forcément initié à l'art.

11 Nous utilisons le terme " image » dans la mesure où ce terme générique est capable de satisfaire l'hétérogénéité sous-tendue par ces œuvres. "Image » désigne ainsi une instance globalisante de la sémiotique visuelle susceptible d'accueillir tous les énoncés en virtualisant toutes les spécificités des pratiques. 
Audrey Moutat | Quand on ne peut croire ce que l'on voit

ferme les yeux. Or, les yeux ne sont-ils pas les attracteurs du regard du sujet percevant ? L'énonciataire s'engage ainsi dans une quête permanente de la relation avec l'autre, d'une communication avec le personnage du « tableau » qui la lui refuse, à travers sa posture. Si le jeu d'ombres attire le regard de l'énonciataire, ce regard ne parvient jamais à rencontrer cette altérité que l'artiste-énonciateur lui refuse.

L'œuvre manifeste ainsi une tension édifiante entre le même et l'autre : cela est dû aux traces du geste pictural de l'artiste manifestées dans l'œuvre (et qui permettent de l'identifier à une photographie de peinture) et à la présence d'éléments de rupture (ou allotopies) ${ }^{12}$ qui engendrent un sursaut perceptif et font douter le spectateur de ce qu'il perçoit (s'agit-il de la photographie d'une peinture ou bien de celle d'un sujet réel ?). Cette rupture de l'isotopie picturale repose sur un différentiel de texture introduit par certaines figures visuelles (les cils semblent être de véritables cils, l'œil est vif et brillant...). Elle touche à l'identité même du genre discursif de l'œuvre et à la nature de l'image. C'est ainsi que le contexte de diffusion de ces œuvres joue un rôle déterminant sur leur perception : lorsque les photographies sont exposées dans une galerie, l'élément dissonant ne s'avère pas nécessairement révélateur de la " tromperie discursive ». Cependant, en situation de " peinture-performance » en galerie ou sur le portfolio de l'artiste où les œuvres côtoient des photographies révélant la technique alors employée, la rupture dans le texte, introduite par le relief en trois dimensions et le rendu de la luminosité, se trouve ainsi résolue.

Arrêtons-nous un instant sur ce jeu véridictoire de la luminosité. Dans son étude consacrée aux tensions de l'énonciation visuelle, Marie Renoue (2001) souligne l'influence de la brillance sur l'étendue spatiale et notamment sur la profondeur : l'intensité de l'éclatant corrélée à une étendue minimale engendre un effacement de la matière picturale au bénéfice de ce qui est extérieur au tableau. Or, dans les œuvres d'Alexa Meade, c'est le mouvement inverse qui se produit : Jaimie ${ }^{13}$ (2010) manifeste en effet une profonde « inertie » qui tend à concentrer le regard du spectateur sur l'aplat créé entre le fond et la figure. Ici, le geste artistique de la représentation semble complètement nié au profit de l'engouffrement d'une figure qui, au lieu de se détacher du fond, s'y noie totalement. Cette indistinction dans le passage entre la figure et le fond se joue grâce aux effets d'ombres produits par l'artiste. Tel est le cas de la majorité des œuvres d'Alexa Meade. Elles manifestent un faible contraste entre le personnage et le fond : ce dernier se présente comme une surface foncée, texturée par les touches de pinceau qui matérialisent un certain relief, une sorte de troisième dimension produite sous l'effet de la macule. Ces zones d'aspérités créent également un jeu de luminosité où les fortes zones d'ombre creusent l'œuvre pour y accueillir la figure. Le contour, limite par laquelle la forme est censée " prendre » et acquérir une certaine consistance pour se détacher du fond, perd sa fonction de clôture de la figure en la connectant et la déconnectant du fond. Dans Natura Morta ${ }^{14}$ (2009), la femme en sous-vêtements,

12 Incompatibilité entre deux unités de signification (Rastier, 1987).

13 Jaimie, 2010, 16»x20» Edition 7.

14 Natura Morta, 2009, 24»x18» Edition 7. 
Audrey Moutat | Quand on ne peut croire ce que l'on voit

assise sur une chaise, tête et regard baissés, se présente comme une figure qui se distingue en tant que telle mais qui ne parvient tout de même pas à s'extraire du fond tant ses contours s'y trouvent dissous. La forme s'individualise donc au sein du fond selon un continuum topologique. Cette structure d'ensemble repose notamment sur une homogénéisation chromatique - mis à part quelques contrastes au niveau des vêtements - qui assure la cohésion de la configuration. Le tenir-ensemble, y compris cette solidarisation constante que l'on voit opérer avec le fond, repose sur le principe du passage qui « consiste à répandre en quelque sorte à côté de l'objet une valeur [une tonalité] qui lui est empruntée, claire ou sombre $»^{15}$. Une telle tension entre connexion et déconnexion de la figure au fond est ainsi rendue possible par l'action de la lumière qui, en produisant des ombres, fait émerger par contrastes des saillances au sein de l'œuvre. Ces « effets-lumière » dessinent des espaces et des formes, gomment l'apparence de certains objets pour en imposer d'autres et créer un relief. Il n'est pas question ici d'éclairage (car nous aurions des indices, sur l'image, d'un point de source lumineuse) mais de lumière interne à l'œuvre elle-même et qui fonctionne comme un régulateur de l'acte perceptif en créant des attracteurs pour le regard. Il s'agit d'un éclairage " débrayé » en ce sens où la lumière crée un artefact visant à oublier la matérialité du support, et notamment sa tridimensionnalité, pour construire un relief interne à la forme peinte.

Pourquoi parler d'« éclairage débrayé » ? Parce que la lumière semble fonctionner en totale autonomie en créant ses propres fluctuations au sein de l'œuvre et en redessinant les contours des objets ; ce qui influe sur leur autonomie et leur identité. Cet éclairage est rendu visible par ses variations sur la surface du visage du personnage, parfois doté de valeur d'éclat (comme dans Jaimie), et faisant apparaître des saillances qui se détachent et accentuent le contraste avec la pénombre du fond. " Modalisatrice des apparences », la lumière exerce des contrastes de luminosité. Elle se veut valorisatrice d'une texture qui n'est pas liée au support, et qui ne résulte donc pas exactement de la coopération des trois instances (support, matière, geste pictural).

\section{De la texture de l'œuvre}

La notion de texture renvoie au rôle de la corporéité dans l'articulation de la signification. Trace du geste de l'artiste, la texture témoigne à la fois de l'intervention du corps de l'énonciateur et des propriétés du support. À la question " pourquoi s'agit-il, selon nous, d'une texture débrayée ? ", nous apportons un second élément de réponse : la nécessité de la texture de composer en procédant à une négation des propriétés matérielles du support. Explicitons ce point.

Selon Jean Dubuffet (1973 :25), « [l']art doit naître du matériau et de l'outil et doit garder la trace de l'outil et de la lutte de l'outil avec le matériau. L'homme doit parler mais l'outil aussi et le matériau aussi ». Et « chaque matériau [...]

15 Lhote repris par Anne Beyaert-Geslin dans « Métamorphoses intermédiatiques (Le bruit de fond de l'image) », Le sens de la métamorphose, Limoges, PULIM, 2009, p. 245 . 
Audrey Moutat | Quand on ne peut croire ce que I'on voit

étant un langage, qui n'en admet aucun autre et ne peut en servir aucun » (Op. Cit. : 23), l'artiste doit s'en conformer et composer avec lui. Or, dans le cas des œuvres étudiées ici, les matériaux sont multiples (murs, corps, objets sont des supports de matières différentes) et convoquent des langages variés. Le matériau suscitant des usages précis, ses propriétés subsument le faire de l'artiste. « [A]ssocié à une pratique, il entre dans un programme d'actions précis dont il prédit la scène prédicative et le modus operandi » (Beyaert-Geslin, 2008 : 102). Dans les œuvres d'Alexa Meade, la pratique picturale est mise en œuvre par l'application, à larges coups de pinceau, d'une peinture associée à une dizaine de composants tels que les produits de beauté et les ingrédients culinaires. La matière ainsi utilisée est donc composite ; le pigment, associé à un liant, se trouve alors mêlé à ces divers "ingrédients ». La production de l'œuvre consiste à recouvrir le support de cette " mixture » tout en déjouant les contraintes liées à la tridimensionnalité et aux textures de ce dernier (peau, tissu, bois, plastique...). Cela implique une modulation du faire : la matérialité de ces supports génère ainsi d’infinies variantes sur le modus operandi. L'artiste se doit parfois de réitérer son geste selon le potentiel d'absorption de la matière par le support, de densifier ses traits et d'épaissir les couches de peinture afin de créer davantage de relief ; et cela, dans le but d'uniformiser le plan de l'expression de l'œuvre et de gommer tout relief propre au support lui-même ${ }^{16}$. Cette uniformité est telle que l'observateur de l'objet artistique ne distingue pas la tridimensionnalité du support mais perçoit un ensemble homogène, une sorte d'aplat sur lequel a été réalisée l'œuvre picturale. Mais le modus operandi ne s'arrête pas là car l'énoncé ainsi obtenu fait ensuite l'objet d'un second débrayage : la photographie de l'œuvre peinte. À une syntaxe manuelle, gestuelle et sensori-motrice succède alors une seconde syntaxe, celle de l'empreinte de la lumière sur une surface sensible.

En creusant un écart avec le réel, la photographie neutralise définitivement le vivant par la superposition d'une seconde couche énonciative. En effet, la dynamique vitaliste de l'installation, aussi infime soit-elle ${ }^{17}$, est virtualisée par l'empreinte photographique qui réactive, voire renforce, le genre pictural de l'œuvre. Prenons l'exemple de Blue print ${ }^{18}$ (2010). Cette œuvre représente un homme, pieds nus, assis dans une posture décontractée, sur un fauteuil situé dans un décor très pauvre. Aucun meuble n'est représenté, hormis le fauteuil sur lequel il est assis. Les murs sont nus, peints en bleu, tout comme le sol, le fauteuil et le personnage lui-même. Des chaussures, bleues elles-aussi, sont disposées, lacets ouverts, à gauche de l'image. Les traces exagérées du geste pictural noient le sujet dans un tourbillon de bleu, en reconfigurant les contours et les traits, et renégocient également la perspective de l'œuvre. Les ombres réelles de l'installation se trouvent dissimulées sous des ombres si-

16 Si relief il y a, c'est uniquement grâce aux effets du geste pictural qui gomme les aspérités réelles du support pour en créer de nouvelles, au sein de la représentation elle-même.

17 Avant d'être saisie par la photographie, l'œuvre est une installation où un sujet vivant peint se tient immobile, dans un décor également peint. Seuls quelques battements de cils ou les mouvements respiratoires trahissent sa qualité d'être vivant.

18 Blue print, 2010, 24»x18» Edition 7. 
Audrey Moutat | Quand on ne peut croire ce que l'on voit

mulées, créées par le jeu des textures et des couleurs de la peinture. Une telle perspective s'avère alors déroutante : elle situe le personnage sur un point de déséquilibre. Il semble glisser sur une pente orientée vers le spectateur et n'a que son fauteuil pour seule accroche. La perspective offerte par la tridimensionnalité de l'installation se trouve définitivement écrasée par la photographie qui permet, paradoxalement, de réaffirmer le genre pictural du premier énoncé : dès lors, la photographie devient celle d'une peinture et celle d'une installation. Geste pictural et empreinte photographique œuvrent donc de concert. Cette nouvelle situation engendre ainsi une confusion entre la « chair du monde » et la « chair de la peinture ». Dès lors, cette réaffirmation du genre pictural par l'empreinte photographique ${ }^{19}$ soulève une tension entre matérialisation et dématérialisation, la première actualisant les propriétés du support matérie ${ }^{20}$ dans l'œuvre, autrement dit la matérialité des éléments peints, tandis que la seconde les potentialise dans le support formel ${ }^{21}$ (en l'occurrence, la texture et l'espace débrayés) afin de servir un projet de représentation des objets du monde. Or, les images qui nous sont proposées au terme de ce double débrayage ont la particularité d'introduire une sorte de terme complexe alliant les deux pôles de cette tension. Car si le travail de l'artiste n'est pas motivé par un projet figuratif contraint par une pression iconisante (représenter des objets du monde de la manière la plus fidèle qui soit), il ne matérialise pas plus les propriétés du support matériel. Ce dernier est en effet dissimulé sous les traces du pinceau visant à aplanir toute disparité de surface afin de créer l'illusion d'un support stable et homogène (celui d'une toile). Quant à la ressemblance iconique, on voit bien que ce n'est pas de cela dont il s'agit : la peinture réalisée à même le référent en exagère les traits, privilégiant la touche picturale qui altère la naturalité du grain de peau. Seule l'identification des objets du monde est ici visée. Ce qui nous amène à penser que le choix des supports et le geste de l'artiste, à l'origine de la structure graphique de l'œuvre et de sa texture débrayée œuvrent en faveur d'un méta-discours sur la peinture elle-même. On peut ainsi y voir une représentation des propriétés d'un support matériel (une toile) et d'un modus operandi (le geste pictural) sur un support formel.

Or, si cette texture débrayée conduit l'observateur à penser qu'il a affaire à la photographie d'une toile, la texture propre au matériau de l'œuvre refait parfois surface et brouille la perception de cet observateur : Blue print (2010) interpelle le lecteur de l'image par une rupture dans la continuité graphique. La texture picturale et la régularité du trait se trouvent altérées par la résurgence des paupières rosées et de l'œil vif et brillant de l'homme peint. L’identification

19 En l'absence de tout contexte permettant de dévoiler les conditions de production de l'œuvre, l'observateur saisit et interprète la photographie comme étant l'image d'une peinture, autrement dit la représentation d'une peinture sur toile.

20 Le support matériel correspond à la matérialité proprement dite du support (argile, papier, granit...).

21 « Le support formel est la structure d'accueil des inscriptions, l'ensemble des règles topologiques d'orientation, de dimension, de proportion et de segmentation, notamment, qui vont contraindre et faire signifier les caractères inscrits » (Fontanille, 2005). 
Audrey Moutat | Quand on ne peut croire ce que l'on voit

de ce détail fait alors douter l'observateur de ce qu'il voit (personne représentée ou personne réelle ?) et du modus operandi de cette œeuvre énigmatique. Selon le groupe $\mu$ (1992), les «familles texturales » peuvent être classées sur la base de deux paramètres, le choix d'un élément et sa loi de répétition. La duplication des éléments produits par le faire pictural garantit, en ce sens, une continuité dans la peinture, une certaine homogénéité et régularité de texture qui va cependant se trouver mise à mal, dans certaines œuvres, par la manifestation d'une matière qui, perçue comme hétérogène, va perturber le mouvement du regard (notamment les cheveux ou l'aspect brillant de l'œil observés dans Shift ${ }^{22}$, 2012). Ces éléments introduisent alors une sorte de dissonance au sein de l'œuvre et entraînent une renégociation du sens : en faisant émerger un sursaut de vie, un mouvement vitaliste dans la peinture, ils donnent ainsi à l'observateur l'illusion d'être face à une œuvre réalisée selon le principe du collage. Cette impression de collage est d'autant plus forte qu'au niveau interprétatif, c'est l'isotopie ${ }^{23}$ picturale qui domine et détermine l'impression référentielle $^{24} \mathrm{du}$ lecteur de l'image. Nous y reviendrons un peu plus loin.

\section{Une ouvre intermédiatique}

Cette rupture au sein de la texture, amorcée par le maintien (ou la résurgence) de la texture propre au support matériel ${ }^{25}$ de l'œuvre, invite à considérer la dualité médiatique de l'image. Comme nous l'avons souligné, Alexa Meade brouille les pistes entre la réalité et sa représentation en laissant volontairement une partie du corps non peinte (cheveux, yeux, mains) avant de fixer le tout dans une empreinte photographique. Dès lors, l'œuvre définitive matérialise une tension permanente entre peinture et photographie. L'image présente ainsi une structure complexe où elle s'avère être à la fois une image de peinture et une simple image photographique ; celle-ci englobant finalement celle-là.

Si nous avons vu que la photographie permettait de fixer la manière dont la peinture entretient un méta-discours sur la praxis picturale ${ }^{26}$, nous verrons également que la peinture fixe les conditions possibles d'une métamimesis photographique. Observons à présent comment les genres se répondent l'un l'autre.

22 Shift, 2012, 160x160 cm Edition $3 \mid 100 \times 66 \mathrm{~cm}$, Edition $5 \mid 75 \times 50 \mathrm{~cm}$, Edition 7.

23 Récurrence d'un même sème rendant possible la lecture uniforme d'un récit. Voir à ce propos Rastier (1987).

24 Représentation mentale qui résulte de l'interprétation d'un texte (Rastier, 1987).

25 En l'occurrence le sujet humain sur lequel l'artiste applique directement sa peinture.

26 Notons par ailleurs quela rupture texturale permetégalement un renouvellement de la plasticité de l'image par l'ouverture de sa dimension haptique (autrement dit le « toucher du regard ») et offre un décrochage vers le réalisme photographique conférant un caractère hyperréaliste à l'œuvre. Cet hyperréalisme est également renforcé par l'adoption d'un cadrage propre à l'énonciation photographique. 
Audrey Moutat | Quand on ne peut croire ce que l'on voit

D'ordinaire, la photographie rétablit le rapport de profondeur avec son sujet via le flou (c'est le principe de la distance focale) ; une pratique qui se trouve ici contrecarrée par le décor peint et par la simulation d'une profondeur fondée sur le principe du passage. En aplanissant la tridimensionnalité du réel, mouvement anticipé par le jeu d'ombres coordonné entre les différents éléments de l'installation, la photographie tient un métadiscours sur la peinture via l'actualisation d'une métamimesis. On pourrait ainsi affirmer, avec Anne Beyaert-Geslin (2009 : 246), que " l'œuvre tient un discours réflexif sur la façon dont [la pratique picturale] rend sensible le monde sensible ».

Comment cela opère-t-il ? Ce principe n'est pas propre à la photographie ; ce que l'on observe, c'est que l'installation est elle-même conçue de façon à préparer l'empreinte photographique. Alexa Meade amplifie son geste pictural en exagérant ses traits expressionnistes et en accentuant l'intensité de sa touche. Le métadiscours porte également sur le genre du portrait où l'on retrouve la figure, la plupart du temps à hauteur de buste, sur un fond coloré uni et sobre.

Mais l'œuvre se veut également être un métadiscours de la pratique photographique comme le montrent la stratégie énonciative du regard (qui impose une manière d'être et d'agir du sujet photographique et de l'observateur de l'image), la pose de certains modèles ou encore le cadrage propre à l'énonciation photographique. L'autoportrait Exposure ${ }^{27}$ (2009) ou encore Blue print (2010) présentent des personnages dont la pose s'avère tantôt sensuelle, tantôt décontractée, avec une nonchalance propre aux protagonistes de certaines campagnes publicitaires. Saisies par le geste de la tradition expressionniste, ces sujets adoptent des poses actuelles, semblables à celles des mannequins des magazines contemporains et offrent ainsi un certain anachronisme.

Quant au cadrage, il se présente comme la trace qui matérialise le geste du corps de la photographe qui construit un champ perceptif autour d'elle. Ainsi que le note Serge Tisseron (1996), repris par Maria Giulia Dondero dans Sémiotique de la photographie (Basso Fossali et Dondero, 2011 : 60), la photographie n'est pas uniquement une façon de prendre possession du monde mais elle permet de laisser un signe de soi-même dans le monde qui atteste de son point de vue : « Le photographe laisse la trace diffuse de sa sensori-motricité [au sein de l']image ». Position que l'on retrouve également chez Denis Roche (1982: 73) : selon ce dernier, la pratique photographique se fonde sur les principes de l'autobiographie et de l'instantanéité car en prenant une photographie, « on photographie ce qu'on a regardé, donc on se photographie soi-même ». Si les traces de cette sensori-motricité ne se matérialisent pas dans le flou (la proximité dans les tons et la récursivité du geste pictural dans la création de la figure et du fond ne le permettant pas ici), c'est dans le regard textualisé de la photographie que nous l'appréhendons, à travers la sélection du cadre du monde : la plongée ou encore le cadrage mettent en exergue cette prise de position de l'artiste en un endroit précis de l'installation pour communiquer, par une mise en abîme, sur la réflexivité de l'œuvre. Tel est le cas de la plongée sur la femme dénudée de Natura morta (2009) qui offre un cadrage intégrant des objets dignes d'une nature morte. Et surtout, il s'agit

27 Exposure, 2009, 16»x20», Edition 7. 
Audrey Moutat | Quand on ne peut croire ce que I'on voit

de choisir le meilleur point de vue qui ne permettra aucune défaillance des ombres pouvant trahir le dessein de la tromperie visuelle.

On note alors, au sein des œuvres d'Alexa Meade, que le regard énonçant déclenché par l'acte photographique (autrement dit la sélection d'un point de vue au sein de l'espace et le cadrage d'un fragment de l'installation) ne peut coïncider avec le regard énoncé au sein de l'installation construite lors de la première séquence énonciative (" peinture-performance »). Et c'est de cet écart que résulte toute la difficulté de la référenciation énonciative. Il est en effet très difficile pour l'observateur de la photographie de l'identifier comme étant celle d'une installation et non celle d'une toile. Alors que l'installation exposée en galerie offre une multitude de points de vue possibles, le regard énonçant de la photographe n'en privilégie qu'un. De ce fait, l'opération de référenciation ne peut conduire à une authentification du véritable genre de l'œuvre. Seule la connaissance de l'ensemble du contexte énonciatif permet de trahir le jeu sur la référence.

Le cadrage trahit la stratégie énonciative adoptée par l'artiste : il renvoie à son propre regard, celui qu'elle veut bien porter sur l'œuvre initiale. En fonction de la sélection opérée par le cadrage sur les éléments intégrés au champ, ou selon l'angle de prise de vue, ce regard confère aux actants de l'œuvre une manière d'être et d'agir (Fontanille et Shaïri, 2001: 88) et détermine, plus largement, le genre de l'œuvre.

À quel système énonciatif avons-nous donc affaire ? Notre position rejoint l'hypothèse de départ de Joseph Courtés (1998) qui, envisageant l'énonciation comme le rapport d'un sujet d'énonciation à son objet (énoncé), considère ce sujet comme l'articulation entre deux actants : l'énonciateur, qui construit son énoncé en convoquant un ensemble spécifique de signes selon une intention particulière, et l'énonciataire, qui interprète cet énoncé selon son propre système de références et de connaissances. Dès lors, l'analyse doit porter son attention sur l'un et l'autre de ces pôles, l'objet s'avérant l'instrument de leur médiation ${ }^{28}$. Doté du statut de signe, l'objet ne peut voir son sens arrêté que dans un contexte d'énonciation donné. Joseph Courtés (Ibid. : 15) envisage ainsi « l'acte d'énonciation comme "actualisation", en tant qu'il produit un objet sémiotique donné ; une fois cet objet mis en circulation (entre énonciateur et énonciataire), on pourra dire que l'énonciation correspond à l'étape sémiotique dite de la "réalisation" ». En effet, l'énonciation est un dispositif articulé en différentes phases, chacune dotée d'un mode d'existence différent. Pour mener à bien son projet communicationnel, l'énonciateur dispose d'un large répertoire de formes sémantiques et syntaxiques qui, dès lors qu'il n'est pas convoqué, restera à l'état virtuel. La production (ou actualisation) de l'énoncé (ou objet sémiotique) consiste à sélectionner, au sein de ce stock, les formes de l'expression et structures discursives les plus adéquates au contenu à véhiculer. L'objet sémiotique ne se réalise véritablement (autrement dit accède pleinement à la signification) qu'au moment de son interprétation par l'énonciataire.

28 L'efficience d'une stratégie énonciative peut ainsi être mesurée en mesurant l'écart entre les intentions sémiotiques d'un énonciateur et les représentations que l'énonciataire se fait de l'énoncé communiqué. 
Audrey Moutat | Quand on ne peut croire ce que I'on voit

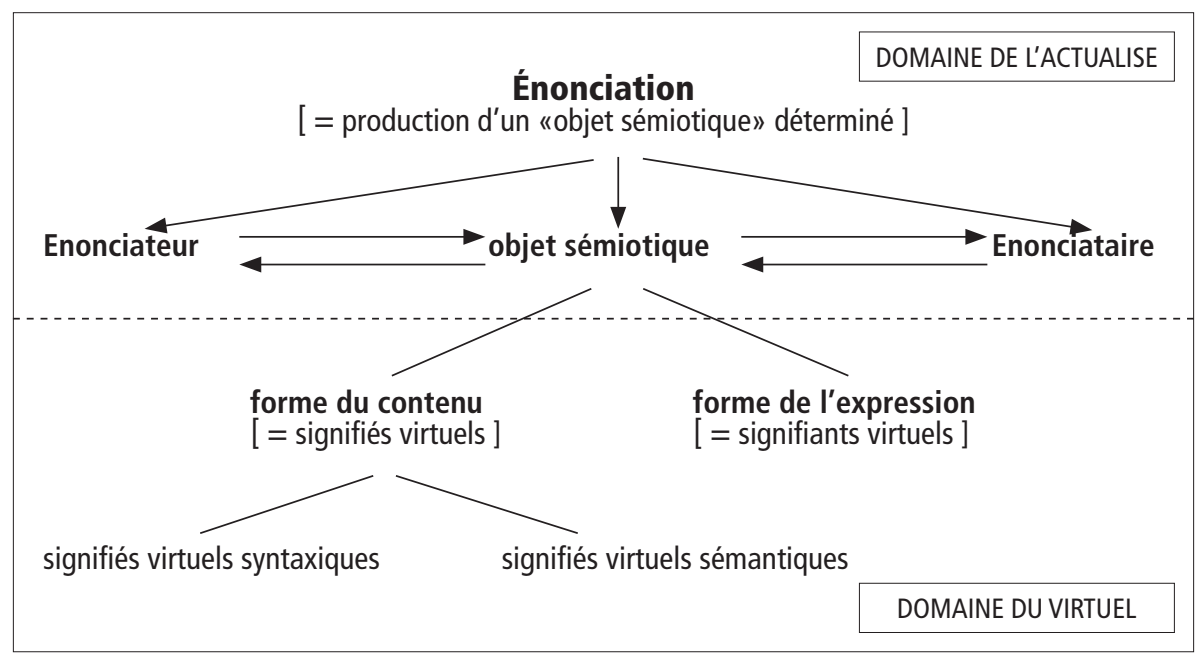

Figure 1. Schéma de l'acte d'énonciation (Courtés, 1998 : 15)

Le cas des œuvres d'Alexa Meade se révèle ici assez subtil dans la mesure où l'objet sémiotique, en l'occurrence l'œuvre artistique, résulte d'un système d'emboîtements énonciatifs. La première situation d'énonciation est celle de la performance-réalisation de l'installation qui actualise un premier objet sémiotique, la " peinture vivante ». La praxis énonciative consiste alors en une amplification du geste propre à la peinture sur toile où la largeur de la touche, le choix des couleurs, le jeu sur les ombres, potentialisent en l'anticipant la seconde situation d'énonciation : la saisie photographique. Un second objet sémiotique débrayé du premier est ainsi créé : la photographie. Cette dernière est donc produite par un enchâssement de situations d'énonciation, la seconde englobant la première. Le premier objet créé, l'installation, présente une matérialité spatiale : tridimensionnelle, elle manifeste une multitude de facettes, de relations entre ses parties inertes et sa partie vivante. Les contours et autres zones d'ombre étant définies conformément à une prise de position dans l'espace programmé (celle que prendra la photographe pour fixer l'œuvre finale), on note que l'énonciation consiste dès lors en la formulation d'un choix, celui d'une facette de l'œuvre au détriment des autres qui seront abandonnées : "Une énonciation donnée est une actualisation qui virtualise du même coup tous les angles de vue disponibles » (Courtés, Ibid. : 34).

En outre, cette stratégie énonciative adoptée par l'artiste nous invite à reconsidérer le principe d'espace photographique défini par Philippe Dubois (1990 [1983]). Selon cet auteur, le langage photographique est articulé par quatre espaces successifs : 1) l'espace référentiel, 2) l'espace représenté, 3) l'espace de représentation et 4) l'espace topologique. Ainsi, la pragmatique de l'énonciation photographique de Dubois situe l'espace référentiel comme point de départ de la réalisation de toute photographie en raison des liens qu'il entretient avec l'espace représenté. Quant à l'espace de représentation, il est le lieu à partir duquel « s'organisent, à l'intérieur du champ, les figures de l'espace représenté » (Op. Cit. : 193). Ce sont ces figures qui, dans un quatrième temps, se confrontent à l'espace topographique du spectateur. 
Audrey Moutat | Quand on ne peut croire ce que l'on voit

Pour Dubois, l'espace représenté et l'espace de représentation constituent " conjointement l'espace photographique proprement dit, les deux extrêmes se rejoignant par leur statut dans leur principe d'extériorité par rapport à l'image elle-même » (Op. Cit. : 197). Or, les photographies d'Alexa Meade, qui relèvent du genre de la photographie plasticienne et non de la photographie d'auteur, montrent au contraire que l'espace photographique doit être élargi aux espaces référentiel et topologique. En effet, le modèle vivant, qui relève de l'espace référentiel, est également le support de l'espace représenté (le modèle peint) dont la composition est modélisée en fonction de l'acte photographique à venir, espace de représentation proprement dit. D'autre part, le cadrage, l'angle de prise de vue ou l'échelle de plan constitutifs de cet espace de représentation sont eux-mêmes déterminés en fonction des dynamiques perceptives que l'artiste préconise pour le futur observateur de l'image (autrement dit l'espace topologique). En effet, la production photographique d'Alexa Meade se fonde essentiellement sur l'instance de réception de l'image, sur la tromperie visuelle de l'observateur. Dès lors, l'espace photographique s'articule dans un rapport complexe entre ces différents espaces, l'espace fédérateur étant l'espace topologique. Ainsi, l'ordre des espaces et leur articulation sur le mode de la succession proposés par Dubois doivent être modifiés en considérant la tension entre les dynamiques de production et de réception de l'énoncé photographique.

Dubois s'inscrit dans une approche génétique qui, en portant sur une pragmatique de la production, considère l'empreinte photographique comme condition de production de la photographie. En effet, selon lui, le dispositif énonciatif s'inscrit dans une perspective purement référentialiste : la photographie comme moment et circonstances de la prise d'image. Or, une telle approche ne peut rendre compte de toute la complexité de l'espace photographique. Elle ne permet pas non plus d'analyser les stratégies manipulatoires engagées par Alexa Meade dans la construction des textes photographiques. L'approche générative que nous préconisons nous conduit à présent à nous interroger sur l'énonciation en tant que manipulation mise en œuvre par la circulation de l'objet/énoncé artistique.

\section{La manipulation énonciative de l'œuvre d'Alexa Meade}

Il s'agit véritablement d'une manipulation car, comme le souligne Joseph Courtés (1998 : 37), « peindre, dessiner, construire, etc. [...] ce n'est pas seulement, du point de vue [...] de l'énonciateur, transmettre un savoir donné, ce n'est pas uniquement un /faire savoir/, mais tout autant un /faire croire/ [...] qui met en jeu un système actoriel et modal toujours extrêmement complexe ».

Nous serions ainsi amenée à penser que la manipulation énonciative orchestrée par Alexa Meade vise à faire adhérer l'énonciataire à un « faire croire être un tableau ». Or ce n'est pas exactement cela. En effet, dans la manipulation énonciative telle que l'entend Joseph Courtés, l'énonciateur a au moins un double rôle énonciatif : d'un côté, il a pour tâche de « faire croire » l'adhérent, de le modaliser positivement en vue de lui faire accepter les propositions et 
Audrey Moutat | Quand on ne peut croire ce que l'on voit

points de vue soumis ; de l'autre, il lui faut empêcher l'opposant de croire en autre chose, par un « faire ne pas croire ». S'il est clair ici que la praxis et le débrayage énonciatif actoriel ${ }^{29}$ adoptés par l'artiste « font croire en un tableau », un tel programme n'est pas mené à son terme. Le jeu de l'illusion se trouve révélé à des degrés variables en fonction de la saillance des indices (texture peau des sujets vivants, brillant de l'œil ou texture des cheveux...). Une fois détectés par l'observateur, ces indices invitent à une relecture de l'image et à une nouvelle interprétation de son genre : le contour des yeux, la texture des cheveux, le regard lui-même ou l'absence de peinture sur certaines zones du corps font partie de cette manipulation énonciative qui, en cherchant à prescrire une appréhension spécifique de l'image, module également le parcours interprétatif de son observateur. L'énonciation instaure ainsi un jeu de cache-cache entre objet, énonciataire et énonciateur en convoquant différentes positions véridictoires : le vrai, le faux, l'illusion.

Dès lors, les photographies d'Alexa Meade mettent à mal le principe de transparence défendu par l'approche représentationniste de certains historiens de l'art tels qu'Elizabeth McCausland qui, dans son article « Documentary photography » (1939), considère la photographie comme un signe transparent qui permet à son observateur d'accéder au réel ${ }^{30}$. Les œuvres de l'artiste ne s'inscrivent pas dans une mimétique et n'entretiennent pas une relation d'exactitude avec la réalité visible. Bien au contraire ! En réduisant la tridimensionnalité de l'espace référentiel par la sélection d'un point de vue spécifique dans l'espace de représentation, l'artiste procède à une déformation du réel qui prive l'observateur de toutes autres perceptions qui lui auraient permis de l'expérimenter pour ce qu'il est (la photographie d'une installation en trois dimensions et non une œuvre peinte en deux dimensions sur une toile). Dès lors, et contrairement à ce qu'avance Susan Sontag (2000), la photographie ne s'avère pas plus innocente ni exacte que ne l'est n'importe quel autre objet mimétique.

En superposant le plan d'expression de l'œuvre à son référent (lequel opère ainsi comme un support d'inscription), Alexa Meade nous invite également à nous interroger sur la question de la référence. La mise en œuvre de la pratique picturale sur un support vivant (à même le corps du référent) dramatise le rapport au monde naturel. La représentation picturale fige le vivant, en lui ôtant le rythme de la vie par la stabilité, par la forme stéréotypée de l'objet pictural.

Une question s'ouvre alors, celle du rôle de la manipulation énonciative sur la scène perceptive, cette dernière étant entendue par Jean-François Bordron (2002) comme une énonciation. Selon le sémioticien, le monde naturel fonctionne comme un système énonciatif qui exprime ses aspects perspectifs dans un plan d'expression, lui-même doté d'une signification à part entière.

29 Alexa Meade délègue, au sein du texte, un actant observateur identifiable à l'énonciataire et lui attribue une position spatiale déterminée, extérieure à l'œuvre.

30 C'est une pensée que l'on rencontre également chez André Bazin (1945). Selon lui, « les virtualités esthétiques de la photographie résident dans la révélation du réel » qui opère par un " transfert de réalité de la chose sur sa reproduction ». Image et modèle ne font alors plus qu'un. 
Audrey Moutat | Quand on ne peut croire ce que l'on voit

En effet, tout objet de perception ne se laisse appréhender que sous certains aspects ; les autres restant dissimulés. À cet égard, nous pouvons nous référer à Merleau-Ponty (1976 : 255) qui, identifiant les mouvements comme les manifestations de l'intentionnalité du sujet de perception, reconnaît à l'objet la faculté de se manifester selon certaines esquisses : " Nos intentions trouvent dans les mouvements leur vêtement naturel ou leur incarnation et s'expriment en eux comme la chose s'exprime dans ses aspects perceptifs ». Dès lors, la perception consiste en la rencontre de deux dynamiques : celle d'un sujet dont les mouvements intentionnels sont motivés par les variations des aspects perceptifs (ou esquisses) de l'objet.

Concernant notre corpus, nous avons vu que si l'image ne change pas, le rapport que le sujet percevant entretient avec elle varie ; ce qui produit une réorganisation sémiotique de l'expérience. Lorsque le spectateur de Portrait of a self-portrait ${ }^{31}$ (2009) appréhende la photographie dans sa globalité, il identifie l'œuvre comme étant la photographie d'un autoportrait d'Alexa Meade, peinture sur toile, inspirée du mouvement expressionniste. Mais une inspection particularisante de l'image, autrement dit la scrutation locale de ses différentes parties, isole les formes les unes des autres et fait émerger les dissonances texturales (la texture des cheveux, l'aspect aqueux de l'œil et la brillance des dents du modèle). Ces dissonances se présentent comme de nouvelles esquisses de l'œuvre et engagent de nouvelles variations intentionnelles du sujet percevant : il concentre alors son regard et mobilise toute son attention sur ces éléments en rupture avec la texture globale de l'image. C'est alors que se produit une variation interprétative qui interroge la nature de l'œuvre elle-même. Le changement textural renégocie le parcours de l'image, introduisant un sursaut perceptif qui, sur le plan interprétatif, amorce une réévaluation du modus operandi et du genre de l'image : est-ce une peinture introduisant deux types de modus operandi (expressionnisme et hyperréalisme ?), une peinture-collage (peinture expressionniste et collage photographique ?) ? Une photographie-collage (photographie et collage avec une peinture expressionniste) ? Cette " allotopie texturale » constitue donc un pivot dans l'image, un seuil critique au-delà duquel son genre peut être recatégorisé sémantiquement. Les œuvres d'Alexa Meade génèrent ainsi une contradiction sensible, un double rapport perceptif lié à une variation texturale.

L'accommodation du regard sur l'ensemble de l'image résulte de son apparente stabilité dans les motifs et la texture picturale globale. Or, l'intensification du regard de l'observateur, qui déplace son point d'attention sur chacune des figures de l'œuvre, fait émerger de nouvelles esquisses, plus ou moins aisément identifiables. Cette variation de l'acte perceptif pourrait être définie en convoquant la sémiotique tensive (Fontanille et Zilberberg, 1998) : dans le cas d'une appréhension globale de l'œuvre, l'étendue du champ visuel de l'observateur est corrélée à un affaiblissement de l'intensité de son regard de telle sorte qu'aucune esquisse dissonante n'apparaisse. Ce n'est qu'une fois que ce rapport intentionnel à l'œuvre varie que la signification associée à l'image évolue : l'étendue du champ visuel se réduit à mesure que l'intensité

31 Portrait of a self-portrait, 2009, 16»x20», Edition 7. 
Audrey Moutat | Quand on ne peut croire ce que l'on voit

et l'étendue temporelles du regard augmentent. La tension au sein de l'œuvre s'intensifie et invite l'observateur à la parcourir avec plus d'acuité dans le but de détecter d'autres éléments de résolution de l'énigme. Si la configuration graphique et plastique de l'image reste inchangée, son contenu varie et cela parce que le rapport entre l'œuvre et l'observateur a lui-même changé. Ces variations tensives soulèvent également un problème interprétatif d'un point de vue véridictoire : ce que le parcours holistique de l'image donne pour vrai (photographie d'une peinture) est mis à mal par la visée particularisante (ce que l'observateur croyait vrai est en vérité faux compte-tenu de la texture manifeste des dents, des cheveux et de l'œil). L'expérience de l'image opère selon un principe de désillusion où l'appréhension de certaines esquisses ouvre une autre scène, un nouvel horizon d'image (Bordron, 2013), celui de la photographie d'un être humain réel. Dès lors, l'acte perceptif, initialement orienté vers un horizon d'image (photographie de peinture), subit une sorte de décrochage vers un second horizon (photographie d'une personne peinte). L'allotopie visuelle met en évidence une nouvelle esquisse de l'objet qui émerge dans la scène perceptive sans parvenir à s’imposer totalement. Elle engage ainsi l'observateur dans un parcours cognitif de l'enquête, partant du soupçon d'un quelque chose d'inhabituel au sein de l'œuvre (soupçon suscité par les éléments de rupture texturale) vers l'apparition de ce qui est véritablement (les cheveux, yeux et dents d'une personne réelle). "Vers » car la saisie du véritable objet de la photographie (la personne peinte) ne pourra jamais se réaliser, la forme ne pouvant s'accomplir intégralement.

Cependant, aucune des deux visées ne trouvera satisfaction faute d'une saisie parfaite : ni la peinture, ni la photographie ne pourront se réaliser entièrement ; la première ne présentant pas tous les traits de conformité du genre (texture atypique des yeux et des cheveux pour une peinture expressionniste), la seconde n'étant qu'amorcée par ces ruptures texturales mais non accomplie (puisque la photographie de l'artiste elle-même ne pourra jamais être perçue en tant que telle).

Notons à cet égard que les œuvres d'Alexa Meade ont la particularité de mettre à mal le concept de représentation : « La représentation suppose qu'il y ait eu une présentation et donc un monde originairement présent qui nous assure que, malgré les jeux et les duplications égarantes des représentations, quelque chose aura été représenté » (Bordron, 2009 : 117). Ainsi, l'image-représentation doit s'approcher le plus fidèlement de ce qui existe déjà. Elle remplit sa fonction initiale (rendre présente la chose absente). Or, en l'occurrence, les photographies s'en écartent : l'horizon, autrement dit ce qui est visé par la photographie (reproduire un modèle existant) est déjà là, incarné dans l'œuvre picturale en tant que substance. En effet, le modèle du portrait et le décor dans lequel il évolue font office de support dissimulé sous la forme de l'expression (touches picturales) qui, en créant une couche sur ce qui est censé être l'horizon du portrait, crée une distanciation avec l'être. Au lieu de présentifier la personne absente, le portrait creuse l'absence de son modèle pour mettre en exergue une pratique artistique (la peinture expressionniste). Par conséquent, l'image n'est pas là pour se rapprocher de cet horizon déjà présent (autrement dit le modèle) mais devient elle-même l'horizon (celui 
Audrey Moutat | Quand on ne peut croire ce que l'on voit

d'une œuvre picturale). En outre, la rupture au niveau des formants texturaux de l'œuvre fait varier le rapport de l'observateur à l'image et à la visée de l'œuvre : cette dernière ne propose plus de représenter ce dont il y a image (photographie d'une peinture) mais vise à montrer (et à questionner) le genre de l'image (peinture/photographie ?). C'est finalement l'introduction de l'allotopie texturale qui pose le problème du déplacement de l'horizon de visée de l'œuvre : ce n'est plus la référence qui importe mais le genre lui-même, lequel se trouve sans cesse renégocié par la tension entre deux horizons d'images. Peinture et photographie coexistent et se confrontent ; la domination de l'une ne pouvant faire oublier l'autre dans la mesure où aucune forme n'est entièrement accomplie.

On pourrait en ce sens noter que la perception de l'image dans un contexte d'exposition sur le mur d'une galerie relève du principe du trompe-l'œil où les variations intentionnelles de l'observateur (ses mouvements de rapprochement et de recul par rapport à l'image, ou encore l'intensité de son regard), suscitées par les variations de contraste et de luminosité de l'œuvre font émerger les changements de textures, révélant ainsi une nouvelle réalité de l'image. Cependant, à la différence du trompe-l'œil traditionnel, où la révélation de ce dernier en tant que tel repose sur l'identification de contrastes (texturaux, lumineux, coloriels) de l'objet avec son environnement immédiat ${ }^{32}$, on note que dans les œuvres ici étudiées, l'allotopie à l'origine du sursaut perceptif et de la réinterprétation de l'ensemble est interne à l'image ; la dynamique du trompe l'œil est, en ce cas, interne à l'œuvre. Dans le cas du trompe l'œil ordinaire, la révélation se fonde sur des différences observées entre l'image elle-même et son support qui reste visible. Or, dans les réalisations d'Alexa Meade, aucune résolution de la tension ne peut véritablement aboutir dans la mesure où l'image et le support coïncident parfaitement et que l'image est censée référer à son support (la peinture est censée représenter le modèle ; or, le modèle est déjà là en qualité de support).

En situation d'installation, la topographie du lieu (la galerie) et de l'œuvre elle-même est propice à la motricité du sujet et permet de renouveler l'objet artistique : la tridimensionnalité annihile les aplats que construit l'angle de prise de vue photographique et permet aussi de révéler la « supercherie énonciative ». Ce que ne peut faire la photographie : en redécoupant l'œuvre dans un cadrage spécifique, elle abstrait cette dernière de tout contexte référentiel et fait apparaître le premier énoncé artistique comme autre (une photographie de peinture et non celle d'une installation).

En outre, si d'ordinaire le trompe-l'œil ménage une surprise intense, car non prévisible, liée à la reconsidération globale de la forme où les nouvelles esquisses perçues remplacent les précédentes, ici, toutes les esquisses (peinture et être humain réel) coexistent au sein d'une même forme sans jamais s'occulter. Dès lors, l'image fonctionne comme un évènement perceptif à part entière.

Dans son article consacré à l'expérience d'objet et l'expérience d'image, Jean-François Bordron (2009 : 121) souligne qu' « [i]l y a dans toute énoncia-

32 Un changement de lumière nous fait soudain comprendre que la fenêtre que nous percevions sur le pignon d'un immeuble est en réalité une fenêtre peinte, différente de la véritable fenêtre située juste à côté. 


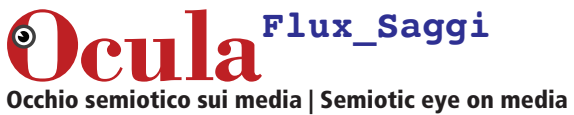

Audrey Moutat | Quand on ne peut croire ce que l'on voit

tion la nécessité d'une double accommodation qui nous porte à entendre à la fois ce qu'elle dit et le fait qu'elle énonce. Par là s'ouvre une troisième dimension de la valeur de vérité qui pourrait se signaler par cette question : l'image est-elle bien ce qu'elle dit être ? ». S'ouvre ainsi la question de la concordance entre la scène de production de l'énoncé et la scène de son interprétation, entendue comme " une expérience qui consiste à retrouver les formes d'une autre expérience dont il ne reste que l'empreinte » (Fontanille, $2004: 265$ ).

Contrairement aux objets (de perception) naturels, les images construites soulèvent des problématiques véridictoires plus profondes. Car une image, telle que celles que propose Alexa Meade, est à la fois un énoncé visuel, produit par un énonciateur qui manifeste une intention de signifier et souhaite agir sur son énonciataire, et un objet de perception (objet sensible qui convoque variablement les sens d'un sujet percevant). Cette perception se trouve fortement contrainte par les choix énonciatifs de l'artiste qui ménage des modes de percevoir particuliers chez l'observateur (tantôt un regard englobant, tantôt un regard particularisant). Et avec cet acte perceptif, c'est un parcours interprétatif de la photographie qui s'engage et permet à l'observateur de comprendre non seulement la complexité de l'œuvre mais également le projet artistique de l'artiste.

Cette interprétation de la photographie par son observateur fonctionne comme une sanction du programme énonciatif mis en place par l'artiste lors de la création de son œuvre. Elle met ainsi en jeu non seulement des modalités véridictoires et épistémiques, mais également une dimension pathémique (influence de l'œuvre sur les états d'âme de l'observateur). L'orientation de la sanction (découverte ou non du subterfuge de l'image) dépend de la visée de l'artiste lors de la production textuelle : en l'occurrence, il s'agit de prescrire des mouvements perceptifs variables capables de faire émerger, à des degrés variables, des écarts perceptifs à l'origine du soupçon sur le genre de l'œuvre. L'objectif de l'artiste vise donc à créer une ambiguïté entre image et réalité, le vrai et le faux, et propose alors un nouveau questionnement (voire une mise à l'épreuve) sur la triade Signifiant/Signifié/Référent. Par ailleurs, ces exemples soulignent toute la dynamique de l'énonciation où la signification est sans cesse remise en question par une négociation permanente entre intentions productives de l'énonciateur et stratégies interprétatives de l'énonciataire.

La stratégie énonciative (emboîtement de scènes énonciatives) adoptée par Alexa Meade dénonce enfin un dernier problème, celui de la reproduction photographique de toute ouvre d'art qui, en proposant une diffusion et réadaptation à des situations différentes, procède à une dévaluation de l'hic et nunc. L'œuvre se voit alors soustraite de sa propre mémoire, devient une image autre qui, en changeant de cadre énonciatif, signifie autre chose et autrement. 


\section{Ocula ${ }^{\text {Flux_saggi }}$ \\ Occhio semiotico sui media | Semiotic eye on media}

Audrey Moutat | Quand on ne peut croire ce que l'on voit

\section{Bibliographie}

Basso Fossali, Pierluigi ; Dondero, Maria Giulia

2011 Sémiotique de la photographie, Limoges, PULIM.

Bazin, André

1958 (1945) « Ontologie de l’image photographique », Qu'est-ce que le cinéma ?

Ontologie et langage, Paris, Les éditions du cerf, pp. 11-19.

Benveniste, Émile

1966 Problèmes de linguistique générale, 1, Paris, Gallimard.

Beyaert-Geslin, Anne

2008 « De la texture à la matière », Éthique et sémiotique du sujet, Protée, vol. 36, $\mathrm{n}^{\circ} 2$ (hors dossier), pp. 101-110.

Beyaert-Geslin, Anne

2009 « Métamorphoses intermédiatiques (Le bruit de fond de l'image) », dans Le sens de la métamorphose (M. Colas-Blaise et A. Beyaert-Geslin dirs.), Limoges, PULIM, pp. 245-246.

Bordron, Jean-François

2002 «Perception et énonciation dans l'expérience gustative, l'exemple de la dégustation d'un vin », dans Questions de sémiotique (A. Hénault dir.), Paris, PUF, « Premier Cycle », pp. 121 - 154.

Bordron, Jean-François

2009 « Expérience d’objet, expérience d'image », Visible, $\mathrm{n}^{\circ} 5$, L'image dans le discours scientifique : statuts et dispositifs de visualisation, Limoges, PULIM, pp. 111-122.

Bordron, Jean-François

2011 L’iconicité et ses images. Études sémiotiques, Paris, PUF.

Bordron, Jean-François

2013 Image et vérité. Essais sur les dimensions iconiques de la connaissance, Liège, Presses Universitaires de Liège, Collection Sigilla.

Courtés, Joseph

1991 Analyse sémiotique du discours. De l'énoncé à l'énonciation, Paris, Hachette supérieur.

Courtés, Joseph

1998 L'énonciation comme acte sémiotique, Nouveaux Actes sémiotiques, ${ }^{\circ}$ 58-59, Limoges, PULIM.

Dubois, Philippe

1990 (1983) L'acte photographique, Paris, Nathan-Labor.

Dubuffet, Jean

1973 L'homme du commun à l'ouvrage, Paris, Gallimard.

Fontanille, Jacques ; Shaïri, Hamid-Reza

2001 «Approche sémiotique du regard photographique : deux empreintes de l'Iran contemporain », Dynamiques visuelles, Nouveaux Actes Sémiotiques, $\mathrm{n}^{\circ} 73^{-}$ 74-75, Limoges, PULIM. 


\section{Ocula ${ }^{\text {Flux_saggi }}$ \\ Occhio semiotico sui media | Semiotic eye on media}

Audrey Moutat | Quand on ne peut croire ce que l'on voit

Fontanille, Jacques

2004 Soma et séma. Figures du corps, Paris, Maisonneuve et Larose.

Fontanille, Jacques

2005 « Du support matériel au support formel », dans L'écriture entre support et surface (M. Arabyan et I. Klock-Fontanille dirs.), Paris, L'Harmattan, disponible sur :

$<$ http://www.unilim.fr/pages_perso/jacques.fontanille/articles_pdf/visuel/ Ecritssupportsconclusion.pdf>

Fontanille, Jacques ; Zilberberg, Claude

1998 Tension et signification, Bruxelles, Mardaga.

Goodman, Nelson

2005 Langages de l'art : une approche de la théorie des symboles, tr. Fr. J. Morizot, Paris, Hachette.

Groupe $\mu$

1992 Traité du signe visuel. Pour une rhétorique de l’image, Paris, Le Seuil.

McCausland, Elizabeth

1939 « Documentary photography », Photo notes, janvier 1939, pp. 6-9.

Merleau-Ponty, Maurice

1976 Phénoménologie de la perception, Paris, Gallimard.

Rastier, François

1987 Sémantique interprétative, Paris, PUF, « Formes sémiotiques ».

Renoue, Marie

2001 «Des tensions dans l'énonciation visuelle (À propos de Peinture $300 \times 325$ $\mathrm{cm}$, 9 juillet 2000, de Pierre Soulages) », Dynamiques visuelles, Nouveaux Actes Sémiotiques, n ${ }^{\circ}$ 73-74-75, Limoges, PULIM, pp. 59-86.

Roche, Denis

1982 La disparition des lucioles. Réflexions sur l'acte photographique, Paris, Éd. de l'Étoile.

Sontag, Susan

2000 De la photographie, Paris, Bourgois.

Tisseron, Serge

1996 Le mystère de la chambre claire. Photographie et inconscient, Paris, Flammarion/Les Belles Lettres.

Audrey Moutat est maître de conférences en Sciences du Langage et en Sciences de l'Information et de la Communication à l'Université de Limoges. Sa recherche porte sur la sémiotique de la perception à laquelle elle a consacré son ouvrage Du sensible à l'intelligible. Pour une sémiotique de la perception (Éditions Lambert-Lucas, 2015). Elle s'étend également aux dispositifs de médiation et de médiatisation du sensible à travers différents objets, tels que les textes, la photographie, le son, la peinture ou encore l'art numérique. 